\title{
ON THE SOLVENCY OF INSURANCE COMPANIES
}

\author{
T. Pentikäinen \\ Helsinki
}

\section{WHAT IS SOLVENCY?}

This report is a contribution to the discussion on the solvency problem, which has been taking place at ASTIN-meetings. In his report in Edinburgh I964 Beard referred to many aspects which are closely connected with the problem. Such aspects are

I. the evaluation of liabilities;

2. the evaluation of assets;

3. the level of the premiums of long term policies and

4. reinsurance.

If all of these are not in order, there is no sense in speaking about solvency. E.g. a solvency margin defined as the difference between assets and the expected value of liabilities would not be a reliable measure of the financial state of an insurance company, if either of these-or maybe both-are not evaluated in a reliable way. The fixing of solvency margins is not an isolated problem, on the contrary it is only part of the security measures which must all be managed at the same time. The ultimate purpose of the security system prescribed by legislation must be to safeguard policyholders and claimants against losses.

However, if the problem of solvency is understood in as wide a sense as is mentioned above, the subject has apparently grown so much that it would be inpracticable to discuss the whole of it at one meeting. That is why it seems to be advisable to limit the scope to the solvency problem "in a narrower meaning", i.e. to the solvency margin question only and to give up items I-3 mentioned above and also partially item 4 and let them be discussed at some other meeting or in some other organisation. The more so because already now in most countries these subjects may be, in a very detailed way, prescribed by Insurance Company Acts and the 
supervising authorities pay a great deal of attention to checking their fulfilment with each insurance company.

Some remarks. Even if items $\mathbf{I}-4$ are not discussed in this paper we are going to give some few comments on them, especially concerning some definitions which are needed later on or concerning some aspects which Beard has mentioned in his report.

r. Mathematical reserves (including the reserve of outstanding claims). In some countries the rules applied provide fairly exact evaluation of outstanding liabilities e.g. as mathematical capital values of future claims. In other countries, in addition to that, some additional amounts are allowed (or even expected) to meet unfavourable future fluctuations and unexpectedly high temporary risks and claims. If "a fluctuation reserve" or "an adjustment reserve" of this kind is included in the mathematical reserves it may be quite correct to take it into account, at least to a certain degree, as a proper part of the solvency margin defined later on.

2. Evaluation of assets. The assets must always be estimated and taken into balance sheets in a cautious way. In fact-due to inflation and other reasons-the actual value of assets may often be much greater than the book-keeping value, the difference being an invisible reserve. It may be reasonable that this difference should be taken into account as a part of the company's actual security margin. E.g. the Finnish Insurance Company Act permits this policy.

An important question to be defined is what is the maximum acceptable book-keeping value of assets. Probably it must be the sales value, with some exceptions concerning long term business.

Beard discussed in his report the problems which can appear when a company is being wound up. It can be quite possible that in certain conditions the sales value falls below the expected market value, thus causing loss. There are, however, some other aspects which counteract this risk and make its omission feasable. It seems to be usual in insurance practice that if a company is on the brink of liquidation, its direction, as a final measure, endeavours to find another company which is willing to take it over. If it succeeds then no sale of assets is needed. There is one aspect which helps to find companies willing to take over. The insurance portfolio represents a certain capital, i.e. the acquisition cost of building up a portfolio can be considerable and the company taking over can calculate that it will be profitable in the future when incomporated in the company's own portfolio. This fact can render it quite reasonable to take over, even if minor deficits appear in the assets or reserves. This reasoning provides, among other things, an adequate level of long term insurance (life assurance etc.), as mentioned in item 3 .

\section{Measuring Solvency}

The definition of solvency can be looked at in two different ways:

a) From the point of view of the management of the company: The continuation of the function and existence of the company must besecured. 
b) From the point of view of the supervising authorities:

The benefits of the claimants and policyholders must be secured.

Apparently definition $b$ is narrower. It does not demand the continuation of the company in all circumstances but also allows it to be wound up. However, also in the case of winding up, the liabilities due to policyholders must be secured either by means of the liquitation of assets and liabilities or the taking over of both by some other company. Definition $\mathrm{b}$ can be approved as a basis of the legal security system. If this is done, then the care of the company's existence can be left to the management of each company, which can be carried out by means of adequate reserves, security loadings of premiums, reinsurance and other means. This means, in principle, that the supervising authorities and the legal security measures shall be restricted to the minimum i.e. to secure the insured benefits only, but otherwise each company shall have freedom to develop its function as it itself desires.

Dr. Pesonen in his paper "Solvency Measurement" (Edinburgh, I7th Congress of Actuaries) expressed the definition of security on these lines as follows:

"The reserve, when the accounts of a certain year are closed, is the amount the company would need in addition to future premiums in order to be capable, with a probability of I - $\varepsilon$, of meeting its present and future liabilities if the company ceased to make new contracts after one year."

The period of one year is the same as the normal accountancy period of the companies. The status of each company can be observed only once a year. If it is then stated to be solvent, the continuation of its activity is allowed for the following year. If the company has not an adequate status, winding up will be immediately enforced if solvency is not re-established in a very short time by means of additional capital, additional reinsurance or by other means.

This definition is so general that it takes into account all kinds of risks without limitation to only some few categories of risks, as is the case in some other definitions.

\section{How CAN SOLVENCY BE TESTED?}

The application of the definition given above provides an analysis of the different risks which can threaten an insurance company. 
I) Random fluctuation of claims. This phenomenon is the object of the study of the theory of risk.

2) The fluctuation of the basic probabilities of the claims and their trends. The cause of fluctuations of this kind may be e.g. weather variations in the field of fire insurance, epidemic diseases in the field of life assurance etc. It is well known that economic conditions have an influence upon the loss ratio of many branches of the non-life business. The period of such fluctuations may be sometimes short (weather) and sometimes long, even several consecutive years (economic depressions).

This phenomenon may be estimated to a certain degree by means of the theory of risk, but to a large extent it must be estimated by very rough methods, on the basis of the behaviour of claim ratios observed in times passed.

3) Losses on investment. Losses of this kind can be caused by many reasons. It can be e.g. the bankruptcy of a loan holder in cases where the valuation of the securities has been too optimistic. Further reasons may be the reduction of the value of equities on the general market, the loss of the value of some real estate caused by some special condition, careless action in the valuation of securities or in holding them etc.

4) Miscellaneous risks. It is probably impossible to record thoroughly all kinds of risks which can affect the status of insurance institutions. Some of them can, however, be mentioned here.

a) Natural catastrophes like hurricanes, earthquakes, landslides.

b) Failure of reinsurance. The reason can be a human error, e.g. the reinsurance of a large risk is omitted or the risk of conflagration is miscalculated. The insolvency of the reinsurer can also give trouble.

c) Emplezzlement or other misappropriation of the company's resources. This risk cannot be completely avoided even by the most competent audit or supervision.

d) Riots, sabotage and other disturbances. Ordinary war risks may be settled by special legislation in various countries and they need not be considered here. We can also presume 
that atomic risks are dealt with by various special measures in an adequate way.

Many of the risks mentioned above are of such a nature that they cannot be reliably estimated in advance, especially risks (4). We must keep in mind that the legal, or any other precautionary measures, can never give absolute safety. If we took into account every, even the utmost improbable, chances of risk, security margins and other measures would become intolerably heavy. All we can do, is try and weigh the risks and security measures on a "common sense" basis, and take into account everything which we know by experience has some realistic probability of occuring and neglect risks of a more theoretical nature, which have small likelihood of ever appearing. The circumstances of course vary very much from country to country e.g. concerning items (a) and (d), which appears to make it impossible to find an international standard for a security margin to cover all cases. Probably the only thing to be done is to develop reinsurance so that it covers as many risks as possible and carefully exclude in companies' insurance contracts responsibility for any risk which could be overwhelming. The duty of the state supervision is to check that these measures are observed in every insurance institution and that the internal control and checking is sufficient to guarantee security in this respect as well.

It seems advisable to leave risks 3 and $4^{--}$-so far they cannot be excluded or covered by reinsurance--to be covered by an appropriate security margin. This will be discussed later on in pa:- 4 . Risks I and 2 cannot be covered by a security margin only; instead a solvent combination of reinsurance and solvency margin is necessary. We are now coming to the question of how the adequacy of such a combination should be tested, i.e. in what conditions a company is secured by a probability $I-\varepsilon$ against the fluctuations $I$ and 2 mentioned above.

From the theoretical point of view probably the most natural way to procede would be to prescribe that the direction of each company be responsible for proving the company's solvency to the supervising authorities by means of actuarial calculations. In principle this is exactly the same method which is already applied 
concerning e.g. the evaluation of assets, evaluation of mathematical reserves, checking of the adequacy of the reassurance etc. The calculation of the security numbers, which is the same as the calculation of the probability of ruin, may be, however, a novel task for most actuaries, besides which there are very many non-life companies which have no actuary at all. This question has been much studied in Finland and Dr. Pesonen has developed methods which seem to be applicable to any company. It is true that a rather exact calculation is possible by means of very complicated methods of the theory of risk (also the periodical fluctuation and trends of the basic probabilities must be taken into account). Electronic computers may often be necessary. In practice this is, fortunately, not too formidable a job, because insurance companies can develop a joint programming for the task and then only some few very simple statistics and pieces of information are necessary as input to the computer; as output we can get the probability numbers demanded. In Finland this method is being experimented with and it also seems to be quite feasible for practical purposes.

Fortunately the necessary probability numbers can in most cases be calculated without any computers - it is possible to develop approximations which give very simple rules. These rules can also be accepted as a basis for the official supervision. Only in those very few cases where the solvency of the company cannot be proved by means of these simple rules has the company the right to show its solvency by means of more complicated methods e.g. by means of computers.

To illustrate the approximation method we can present as an example a formula which is much used in Finland for similar purposes. In fact the ruin probability $\varepsilon$ depends on the other variables and distribution of the theory of risk as follows

$$
\varepsilon=F\left(U, P, S_{M}(z), \lambda\right)
$$

where $U$ is the solvency margin, $P$ the premium income on the company's own retention, $M$ the maximum net retention applied for a single claim, $S_{M}(z)$ the distribution function of the size of one claim, which depends on the reinsurance and on the maximum net retention $M$, and $\lambda$ the safety loading included in the premiums. This equation can be expressed by the well-known generalised 
Poisson function, where the fluctuations and trends of the basic probabilities must also be taken into account. It seems probable that this equations can, for most actual cases, be approximated by a formula as follows

$$
U_{\min }=a P+b \sqrt{M P}
$$

where the equation is solved in respect of $U$ after fixing $\varepsilon$ at some appropriate level and $a$ and $b$ are constants. The first term of the formula covers the fluctuation of the basic probabilities. If, from experience, for example the oscillation and trend (in short periods) of the basic probabilities can be expected to be say 30 percent and if normally the safety loading $\lambda$ is $\approx 0$.I, the constant $a$ may be $0.3-0 . \mathrm{I}=0.2$. The second term covers the pure random fluctuations (category I above) and the constant $b$ depends, among other things, on the chosen ruin probability $\varepsilon$. Often a value $b=2$ or 3 may be appropriate. This formula was presented by the author in the ASTIN Bulletin Vol. II, Part I, Jan. I962 (formula (I8)) and earlier in Skandinavisk Aktuarietidskrift I952. Dr. Pesonen has later on further developed it, replacing the second term by a function $M y(v)$ where

$$
v=\frac{(\mathrm{I}+q) P}{M}
$$

the transformed expected number of claims and $y(v)$ a function representing the excess of the number of claims which can be ready tabulated. The constant $q$ takes into account the changes of the basic probabilities. For larger numbers of $v$ the Pesonen formula gives about the same results as formula (2) whereas for small numbers it gives a more accurate approximation.

Formula (2) is probably suitable for most companies. However, in special conditions, for example when a non-proportional reassurance is used, it must be replaced by other formulas. Also the constants $a$ and $b$ depend on the special conditions of each company and may vary considerably.

We will not discuss here any further details concerning solvency testing, instead we refer to the papers mentioned above and especially to Dr. Pesonen's paper presented to the r 7 th International Conference of Actuaries in Edinburgh and to his reports at this 
colloquium. I think his methods and his principal lines of study are worth attention.

We see that in solvency testing in fact there are two free variables, the solvency margin $U$ and the maximum net retention $M$ (and of course also generally speaking the form of reassurance etc). If the actual margin is small, then also the maximum net retention $M$ must be small and vice versa. If only the risk categories I and 2 above are taken into account, they, in principle, do not fix any absolute minimum amount for the security margin, they only link these two variables to each other by means of the general security equation (I), which expresses the Pesonen solvency definition by means of a formula. An appropriate way of proceding may be first to fix some suitable minimum standard as a solvency margin. For this purpose not only the risk categories $I$ and 2 but also all others mentioned above must be taken into account. We shall discuss this question in par. 4 . When a minimum for the solvency margin is arrived at, then it is left to the reassurance to secure, finally, the solvency of the company. To test this the formulas and methods mentioned above, or other similar methods, are available.

\section{Solvency Margin}

When speaking of the solvency margin we understand, as mentioned above, the difference between the actual assets and liabilities of the company. There are reasons, as shown in the previous paragraph, for setting some minimum amount as the solvency margin in the legislation. Also the question of establishing some international standard has been discussed, as is well known. Such standards can probably be motivated even though we must always keep in mind that the solvency margin is only a part of the general solvency problem and the existence of an actual solvency margin exceeding the standardised minimum does not by any means alone guarantee the company's solvency.

To find a rule for the minimum amount of the solvency margin all the risks mentioned in paragraph 3 must be taken into account and the margin ought to be, with a large probability, sufficient to cover the risks mentioned in regard to reassurance and other safety measures and arrangements discussed above. We start again from 
the items I and 2 concerning random fluctuations and the fluctuation of the basic probabilities.

By means of the theory of risk it is possible to calculate, or at least estimate, for each actual case a solvency margin $U$ by means of the formula (I). We shall not present the formulas in detail and refer instead to publications mentioned above. We only state here that for actual computations it is necessary to know, or to detine, for example the reinsurance method, the maximum net retention $M$ and to have some idea of the magnitude of the safety loading $\lambda$. Also the ruin probability $\varepsilon$ must be fixed. To get a universal measure, which would be applicable to all companies, it is advisable to compute numerous examples on the basis of actual risk distributions and the conventional methods of reinsurance and maximum net retentions and make different assumptions concerning $\lambda$ and $\varepsilon$. An attempt at such a study - in practice very comprehensivewas made in Finland when the new Insurance Company Act was prepared in 195I-I952 (the study exists completely only in the Finnish language, but some main points are published in the article mentioned above).

It is apparent that nowadays, when electronic computers are available, the corresponding computations could easily be enlarged and done in a more efficient and accurate way.

The study mentioned above showed clearly that the solvency margin $U$ is very largely dependent on the assumption concerning the safety loading $\lambda$. In the figure we have shown the two main types. Figure I shows the case where $\lambda$ is positive and fig. 2 shows the case where $\lambda$ is zero or negative.

In most actual cases the safety loading $\lambda$ is positive, but there are also cases where $\lambda$, at least temporarily, can be non-positive (due to variations of the basic probabilities, competition, excessive management expenses etc.). Because the legal margins must be constructed especially to cover the weak cases, it seems to be appropriate to preassume $\lambda$ non-positive. This means that the solvency margin must in some way or other be dependant on the size of the company, which implies some increasing function of the premium income $P$, perhaps having the same shape as shown by formula (2).

On the other hand the study showed that the results were not 
very dependant on the assumption concerning the risk distribution $S_{M}(z)$, if the maximum net retention $M$ was not very large. This means that if by insurance the top risks are cut out, the remaining risk distribution on the company's own retention is less affected by the total ruin probability numbers than was previously expected. This does not hold good of course if the maximum $M$ is large, but we have definitely preassumed that the question of adequate reinsurance is already settled.

From the extensive studies the following rule (converted into English pounds) was obtained

$$
\begin{aligned}
& U=£ \mathrm{I} 3000+0.2 P \text { for } P \leq £ 270000 \\
& =£ 40000+0 . I P \text { for } P>£ 270000
\end{aligned}
$$

$P$ here being the gros premium income of the company.

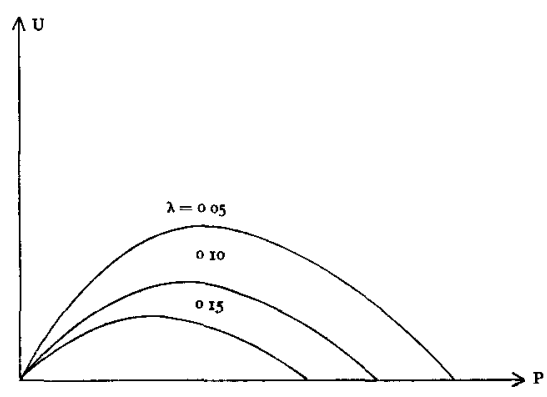

Fig. I

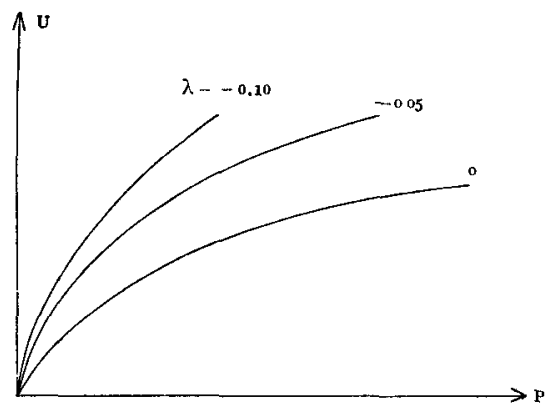

Fig. 2

This rule is very similar to that applied in the United Kingdom, where the margin is Io percent of the premium income, subject to a minimum of $£ 50$ ooo.

On the other hand in most countries the minima of the security margin are fixed sums and do not depend, contrary to formula (3), on the size of the company. It seems to the author that dependence on size is more appropriate. It seems to be very difficult to find a fixed amount which is neither too small for large companies nor too large for small ones, and many of the risks are apparently apt to cause larger losses for larger portfolios.

As yet we have considered rule (3) mainly in terms of random fluctuation and the fluctuation of basic probabilities. However, other kinds of risks, mentioned in paragraph 3, must also be taken 
into account. Varied opinions exist as to whether for each category of risk, for example losses on investment etc. a separate security margin should be prescribed or whether it is enough to have a joint margin for all kinds of risks. In regard to the very small probability of large losses (assuming the supervision of the companies to be adequate, as mentioned in paragraph 2) it may be sufficient to have only one joint security margin, which is intended to be used in cases of emergency for all losses. This means, in other words, that we rely on the great probability, that, for example, a very large random loss and a very large loss on investments can never occur in the same year. The demand for several margins, or security funds, or reserves would, in practice, be unnecessarily burdensome for the companies and would give rise to considerable extra cost if such had to be collected and maintained. Of course in special circumstances, or for special branches, extra precautions can be motivated. For example in many countries a special "security fund" is required for life assurance to cover the risk of future decrease of the rate of interest or other deterioration of the basis of calculation. Such security funds are, however, "semi-obligatory", which means that the non-existence of a security fund does not cause the winding up of the company. Only a compulsory allocation of some portion of the profit may be prescribed in the legislation when the security fund is less than some prescribed minimum amount. We are not here considering these special semi-obligatory funds.

When the special risks mentioned in paragraphs 2 (items 3 and 4 ) and 3 are studied, it becomes apparent that some of them are also of the kind where the amount of possible loss depends on the size of the company. Even though the fixing of a margin for these very heterogeneous risks is extremely difficult and experience in different countries may vary very much, it seems to the author that the margin mentioned above is sufficient even for these difterent risk moments. At least the Finnish committee preparing the Insurance Company Act from the experience in Finland and in some other countries which was available to it came to the conclusion that this formula is sufficient to cover the actual need. Of course a comprehensive discussion on an international basis would be highly desirable to collect all the experience available 
and to fix a suitable minimum standard for the solvency margin. The author's opinion is, that the British and Finnish rule would be applicable at least as a basis for further discussion. In addition to this a method of testing solvency in general, concerning reinsurance and other security measures is needed as presented in par. 3 . 